\title{
Incidencia de las herramientas web 2.0 en el proceso de aprendizaje del bachillerato en la asignatura de historia
}

\author{
Incidence of web 2.0 tools in the high school learning process \\ in the subject of history
}

\author{
Luis Tobías Perez Santana ${ }^{1}$ \\ Francisco Xavier Dillon Pérez ${ }^{2}$ \\ Johanna Cecilia Villavicencio Pazmiño ${ }^{3}$
}

\begin{abstract}
RESUMEN
Las tecnologías de la información y comunicación constituyen un elemento fundamental en el presente trabajo que tuvo como objetivo determinar la influencia de las herramientas web 2.0 en el proceso de aprendizaje de los estudiantes de bachillerato en la asignatura de historia en una institución educativa de la ciudad de Esmeraldas-Ecuador. La investigación fue fundamentada bajo un paradigma cuantitativo, con un alcance en sus resultados de tipo descriptivo, exploratorio y correlacional. La muestra de estudio fue de 96 estudiantes de bachillerato de una institución educativa que oferta la especialidad de bachillerato internacional. Una de las principales conclusiones obtenidas ha permitido confirmar la hipótesis de que el uso de las herramientas web 2.0 inciden en el proceso de aprendizaje de la asignatura de historia en la espacialidad de bachillerato internacional de la población estudiada.
\end{abstract}

Palabras clave: Bachillerato internacional, herramientas web 2.0, historia, proceso de aprendizaje tecnologías de la información y comunicación.

\begin{abstract}
Information and communication technologies constitute a fundamental element in the present work that aimed to determine the influence of web 2.0 tools in the learning process of high school students in the subject of history in an educational institution in the city of Esmeraldas-Ecuador. The research was based on a quantitative paradigm, with a descriptive, exploratory, and correlational scope in its results. The study sample consisted of 96 high school students from an educational institution that offers the international baccalaureate specialty. One of the main conclusions obtained has allowed to confirm the hypothesis that the use of web 2.0 tools affects the learning process of the history subject in the international baccalaureate spatiality of the population studied.
\end{abstract}

Key Words: International Baccalaureate, web 2.0 tools, history, information, and communication technologies learning process.

\footnotetext{
${ }^{1}$ Universidad Tecnológica Indoamérica. Estudiante de la Maestría en Educación mención en Pedagogía en Entornos Digitales. Quito, Ecuador. Correo electrónico: abluisperez@gmail.com. ORCID: https://orcid.org/0000-0002-9046-0945

${ }^{2}$ Universidad Tecnológica Indoamérica. Docente de la Maestría en Innovación y Liderazgo Educativo y de la Maestría en Pedagogía con enfoque en Formación Mediada. Quito, Ecuador. Correo electrónico: franciscodillon@uti.edu.ec. ORCID: http://orcid.org/0000-0002-8776-3435

${ }^{3}$ Universidad Tecnológica Indoamérica. Estudiante de la Maestría en Educación mención en Pedagogía en Entornos Digitales. Quito, Ecuador. Correo electrónico: johav21984@gmail.com. ORCID: https://orcid.org/0000-0002-1121-4157
}

Doi: 10.15517 WL.V1611.45634

Recepción: 12/10/2020 Aceptación: 20/12/2020 


\section{Introducción}

Las Tecnologías de la Información y la Comunicación (TIC), constituyen un referente importante en el fortalecimiento del sistema educativo, por lo tanto, se deben considerar como recursos amigables con la capacidad de modificar los paradigmas pedagógicos actuales. Bajo este contexto la Oficina Regional de Educación para América Latina y el Caribe menciona que: "La experiencia de incorporación de tecnologías en los sistemas educativos de América Latina y el Caribe en los últimos veinte años ha mostrado poco efecto en la calidad de la educación" (Organización de las Naciones Unidas para la Educación la Ciencia y la Cultura 2013, 8).

La Organización de las Naciones Unidas para la Educación, la Ciencia y la Cultura, UNESCO, ha propuesto a sus estados miembros el desarrollo de políticas públicas que aprovechen el potencial de las TIC a favor del desarrollo de la educación con el objetivo de que estas tecnologías contribuyan al mejoramiento sistémico de la misma (Organización de las Naciones Unidas para la Educación la Ciencia y la Cultura 2013, 16)

Los autores Sampedro y Marín (2015) manifiestan que es importante implementar herramientas Web 2.0 en el sistema educativo ya que estas permiten juntar la enseñanza tradicional con el uso de las herramientas digitales tales como redes sociales, wikis, blog entre otros, para lo cual los docentes como agentes educativos formales, deben adquirir destrezas y prepararse respecto al uso y desarrollo del conocimiento tecnológico (Sampedro y Marin 2015, 47,48).

Hoy en día varios países latinoamericanos cuentan ya con estudios relacionados al uso de las herramientas web 2.0 y el proceso de aprendizaje, por ejemplo: como lo señala el informe de la Unesco en el año 2013, en la década de los años noventa, naciones como Costa Rica, implementaron el: "Plan de Informática en Educativa”, Chile con el: “Centro de enlaces". Es pertinente referirse en los últimos años al: "Plan Ceibal" en Uruguay en el mismo orden, se desarrolló en Argentina, el programa "Conectar Igualdad", en Colombia, destaca el programa: "Colombia aprende" y en el caso del Perú, con su iniciativa: "Una laptop por alumno", se debe mencionar que, estas iniciativas de tipo nacional y regional implican un aporte económico hacia el sector tecnológico que se justifica con el aumento de la alfabetización digital. (Organización de las Naciones Unidas para la Educación la Ciencia y la Cultura 2013, 21,22) 
Cuando se refiere al uso de las Tics, no se puede ignorar la importancia que estas tienen dentro de la nueva sociedad del conocimiento y que de una u otra manera obligan a que la educación se adapte a las exigencias de un contexto cada que se encuentra en constante transformación. En aspectos técnicos este impulso es muy claro donde elimina las barreras del espacio y tiempo, facilita el aprendizaje y la comunicación, los canales son inmediatos y permite desarrollar nuevas tecnologías metodológicas para el aprendizaje. (M. Rodriguez 2009). Para conocer más de cerca el uso de las tecnologías de la información y comunicación en el proceso de aprendizaje a continuación se plantea metodológicamente algunos temas y subtemas de referencia diseñados en la siguiente red conceptual. (Ver Figura 1)

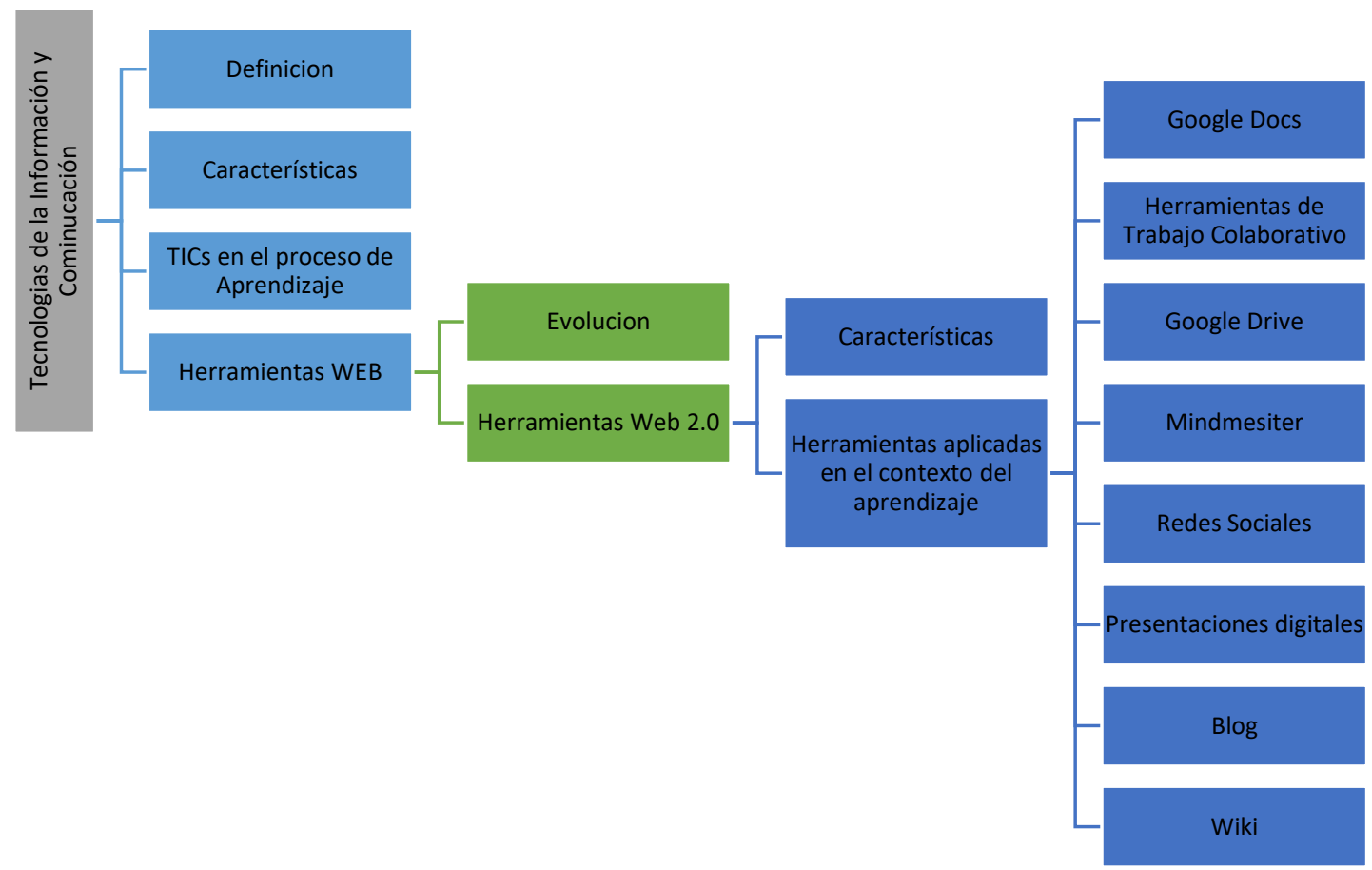

Figura 1. Red conceptual del campo de estudio.

Fuente: Elaboración propia

A continuación, se describen algunos de los recursos y acciones que deberían ser implementados previo la incorporación de las tecnologías en el ámbito educativo: 1) Dotación en las instituciones de sala de informática suficiente y funcional. 2) Capacitación docente en el uso didáctico de las nuevas tecnologías para innovación pedagógica. 3) Creación de redes sociales por áreas para compartir y retroalimentar 
proyectos. 4) Revisión y ajuste de los currículos y proyectos de aula Alfabetización en el uso de las Tics a toda la comunidad educativa (M. Rodriguez 2009).

En cuanto a los procesos de aprendizajes hay factores que inciden en la capacidad de aprender de un sujeto (Aithal y Kumar 2016, 662,663). Por aquello no es suficiente que el estudiante memorice todos los contenidos que recibe en el aula de clases, sino que, esta información, debe comprenderla, analizarla juzgarla y evaluarla para estar en condiciones de aplicarla por lo tanto es muy claro que no existe un único proceso de aprendizaje (Yanez 2016, 78). (Ver Figura 2)

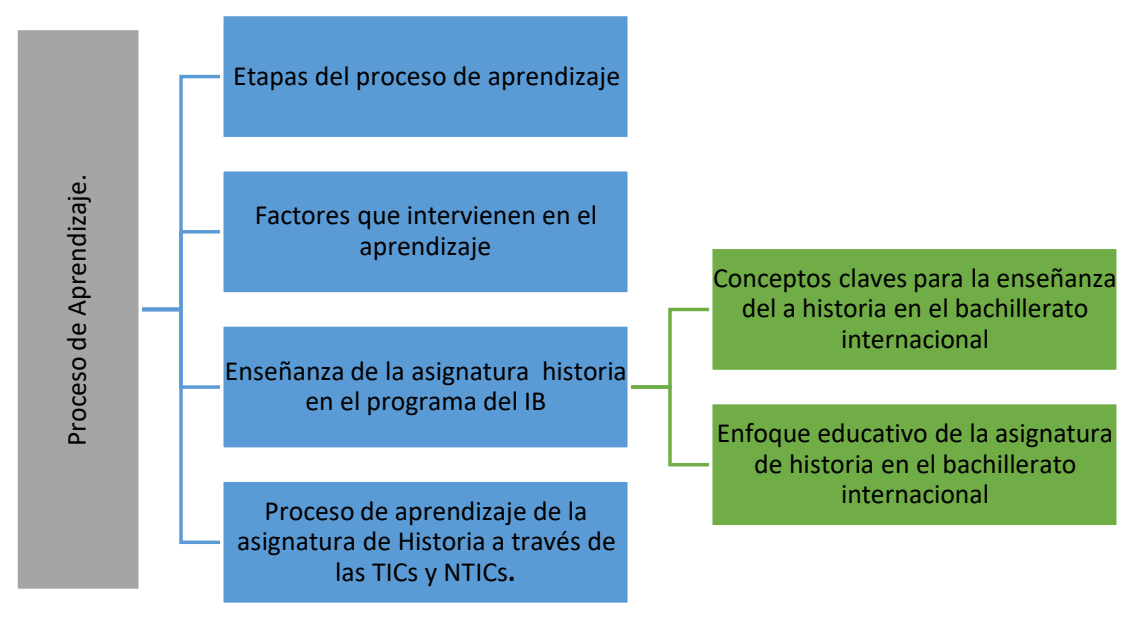

Figura 2. Red conceptual del objeto de estudio.

Fuente: Elaboración propia.

En relación con la asignatura de historia es importante considerar que es una disciplina dinámica, debatida y basada en pruebas que implican un apasionante contacto con el pasado; además, se concentra en conceptos históricos clave, tales como: cambio, causa e importancia. Además, esta conlleva a la exploración y fomenta el sentido de la indagación; también es interpretativa, ya que da lugar a la consideración de múltiples perspectivas y a la pluralidad de opiniones. El bachillerato internacional utiliza estrategias propias de evaluación la cual favorecen una comprensión más profunda de la naturaleza del ser humano y del mundo actual (Organización de Bachillerato Internacional 2017, 6).

La importancia de las TIC hoy en día son un factor fundamental para identificar la incidencia de las herramientas Web 2.0 en el aprendizaje de los estudiantes, para lo 
cual se ha planteado la siguiente interrogante: ¿Cuál es la asociación entre las herramientas web 2.0 y el aprendizaje de la asignatura de historia en una muestra de estudiantes de bachillerato internacional?; sus causas y efectos, se analizan a continuación (Ver Figura 3)

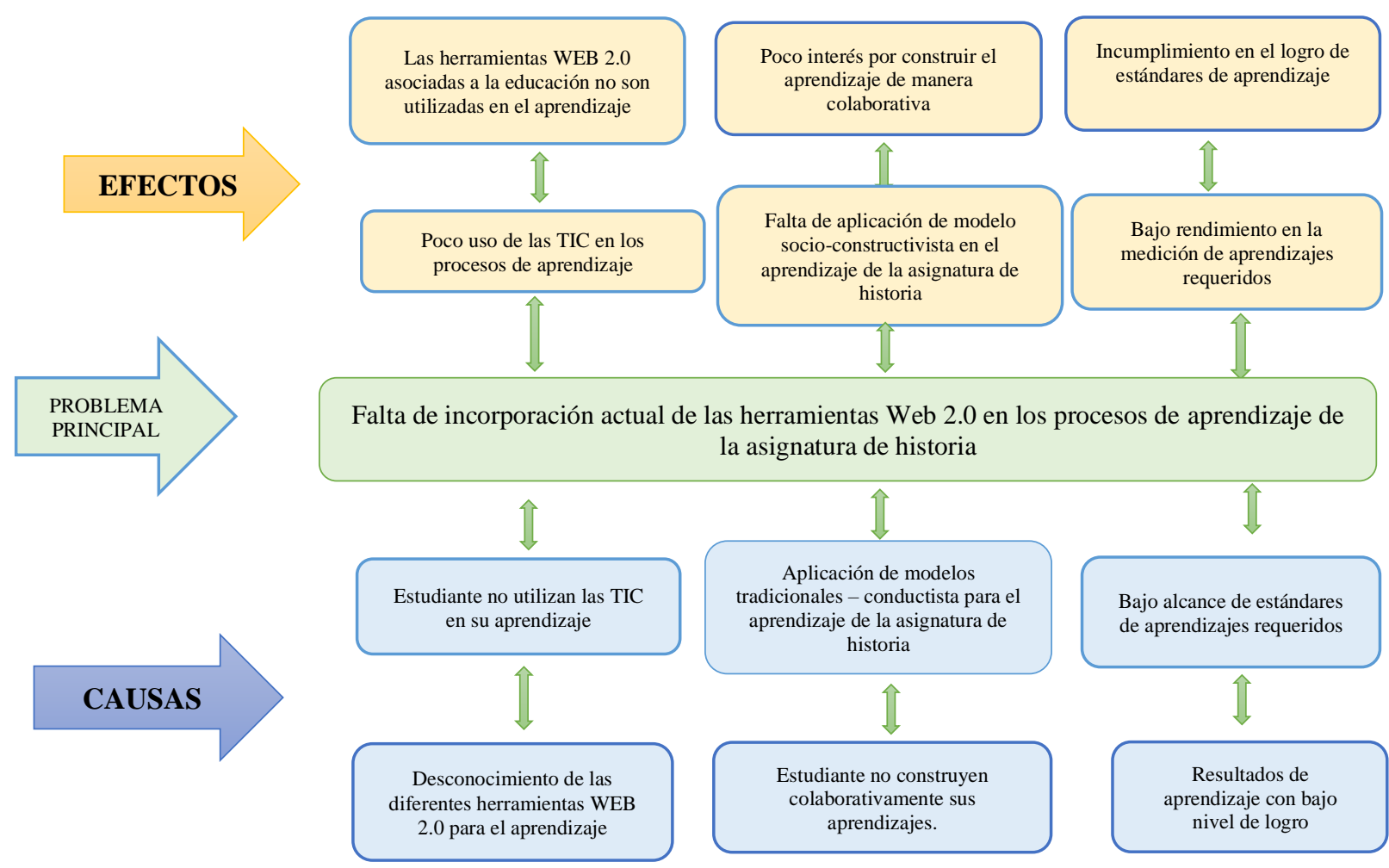

Figura 3. Árbol de problemas

Fuente: Elaboración propia

\section{Metodología}

\section{Objetivo e hipótesis}

El objetivo principal de esta investigación fue determinar la asociación del uso de las herramientas Web 2.0 en el proceso de aprendizaje de los estudiantes de Bachillerato en la materia Historia. Para esto, se plantearon dos hipótesis de trabajo:

- H1 (Hipótesis Alternativa): El uso de las herramientas Web 2.0 inciden en el proceso de aprendizaje de la asignatura de historia del bachillerato internacional de la población investigada. 
- Ho (Hipótesis Nula): El uso de las herramientas Web 2.0 no inciden en el proceso de aprendizaje de la asignatura de historia del bachillerato internacional de la población investigada.

\section{Enfoque de la investigación}

La investigación se fundamentó en un enfoque basado en el paradigma positivista (cuantitativo), el cual representa el conjunto de procesos desarrollados de una forma secuencial (Hernández, Fernández y Baptista 2010, 601); considerando las variables de estudio (proceso de aprendizaje en la asignatura de historia como variable independiente y, herramientas web 2.0 como variable dependiente), la modalidad de investigación planteada es básica, en virtud de que el investigador conoce el problema de estudio y estará en la facultad de producir un nuevo conocimiento que aporte a la comprensión del mismo (Maldonado 2018, 20); además, se ha realizado una investigación de tipo descriptivo y correlacional entre el objeto y campo de estudio (variable dependiente y variable independiente), para de esta forma conocer la relación o grado de asociación del contexto investigado; una vez conocida esta relación, se procedió a cuantificar y analizar su vinculación para así conocer la incidencia entre ellas (Hernández, Fernández y Baptista 2010, 81).

\section{Población}

Para llevar a cabo esta investigación se consideró una población de 111 estudiantes matriculados en el segundo y tercero de bachillerato, de la Unidad Educativa Fisco Misional Juan XXIII, (colegio de bachillerato internacional) de la ciudad de Quinindé (Esmeraldas-Ecuador), los mismos que corresponden a los dos años de estudios del bachillerato internacional. Esta población se encuentra en un estrato socioeconómico medio, con mediano acceso a las TIC y a recursos digitales y multimedia; además, se encuentra caracterizada por una autoidentificación étnica principalmente mestiza.

De la población total de estudio, se ha seleccionado 96 estudiantes (29 varones y 67 mujeres) las cuales fueron encuestadas respecto a las variables de estudio (herramientas web 2.0 y su aplicación en la historia). Para seleccionar a esta población, se realizó un muestreo probabilístico de tipo aleatorio simple, el cual garantiza que todos los elementos de la muestra tengan las mismas probabilidades de ser seleccionados lo que a su vez permitió dar una mayor representatividad al momento de analizar los datos 
obtenidos, en este estudio, el uso de una muestra probabilística, es esencial en el diseño de investigación para correlacionar las variables (Hernández, Fernández y Baptista 2010, 177).

\section{Instrumentos de investigación}

El instrumento de la investigación fue un cuestionario de 12 preguntas que se aplicó a la muestra de estudio seleccionada. Para determinar la determinar la fiabilidad del instrumento, se procedió a realizar una validación a través del juicio de expertos, esta fue realizada por dos expertas en innovación pedagógica y una experta en currículo educativo. Luego de su validación, se realizó el diseño del instrumento de manera virtual a través de la herramienta formulario "Forms" de la aplicación "Google drive", para lo cual también se realizó una prueba piloto considerando al diez por ciento de la población que se iba a encuestar; como resultado se obtuvo el instrumento es "muy confiable" tal como lo demuestra el resultado del Alfa Cronbach (0.9149).

\section{Análisis de datos}

El cuestionario de 12 preguntas de selección múltiple con única respuesta consideró la incorporación de preguntas que midan tanto la variable independiente como dependiente y fue aplicada a la muestra de estudio seleccionada. Se utilizó los resultados de las preguntas 4 y 6 para confirmar la hipótesis afirmativa a través de una prueba de correlación de Chi cuadrado; mientras que, las preguntas 1, 2, 3, 4, 5, 6, 7, 8, 10, 11 y 12 , han sido utilizadas para realizar un análisis correlacional de Pearson permitiendo de esta manera, dar un soporte adicional a los resultados obtenidos en la prueba del Chi cuadrado utilizada inicialmente.

\section{Resultados}

Para comprobar las hipótesis planteadas, se procedió a ingresar los datos obtenidos de la encuesta en el software IBM SPSS Statitics Subscription compilación 1.0.0.1406; los datos utilizados en la comprobación de la hipótesis fueron dos preguntas relacionadas a los enfoques de la enseñanza de historia basados en la pedagogía del bachillerato internacional y la otra, sobre el interés mostrado por los estudiantes en el aprendizaje que generan los contenidos propuestos por el bachillerato internacional. (Ver Tabla 1); el 
recuento de las respuestas esperadas fue analizado con la finalidad de confirmar o descartar la hipótesis nula o alternativa una se procesaron las respuestas y se pudo calcular estadístico de chi cuadrado (Ver Tabla 2).

Tabla 1. Análisis de casos de Chi cuadrado

\begin{tabular}{|c|c|c|c|c|c|c|}
\hline \multicolumn{7}{|c|}{ Resumen de procesamiento de casos } \\
\hline & \multicolumn{6}{|c|}{ Casos } \\
\hline & \multicolumn{2}{|r|}{ Válido } & \multicolumn{2}{|r|}{ Perdidos } & \multicolumn{2}{|r|}{ Total } \\
\hline & $\mathrm{N}$ & Porcentaje & $\mathrm{N}$ & Porcentaje & $\mathrm{N}$ & Porcentaje \\
\hline 6. ¿Considera usted, que los enfoques de la enseñanza & 96 & $100,0 \%$ & 0 & $0,0 \%$ & 96 & $100,0 \%$ \\
\hline \multicolumn{7}{|l|}{ de historia abarcan los principales valores en los que se } \\
\hline \multicolumn{7}{|l|}{ basa la pedagogía del bachillerato internacional? } \\
\hline \multicolumn{7}{|l|}{ 4. ¿Cree usted que los contenidos propuestos por el } \\
\hline \multicolumn{7}{|l|}{ Bachillerato Internacional para la asignatura de historia } \\
\hline generan un interés en el aprendizaje? & & & & & & \\
\hline
\end{tabular}

Fuente: Elaboración propia

Tabla 2. Recuento esperado de Chi cuadrado

Tabla cruzada 6. ¿Considera usted, que los enfoques de la enseñanza de historia abarcan los principales valores en los que se basa la pedagogía del bachillerato internacional?

4. ¿Cree usted que los contenidos propuestos por el Bachillerato Internacional para la asignatura de historia generan un interés en el aprendizaje?

\begin{tabular}{|c|c|c|c|c|c|c|c|}
\hline \multirow[t]{2}{*}{ Recuento } & & & \multicolumn{4}{|c|}{$\begin{array}{c}\text { 4. ¿Cree usted que los contenidos } \\
\text { propuestos por el Bachillerato Internacional } \\
\text { para la asignatura de historia generan un } \\
\text { interés en el aprendizaje? }\end{array}$} & \multirow[t]{2}{*}{ Total } \\
\hline & & & $\begin{array}{c}\text { Algunas } \\
\text { Veces }\end{array}$ & $\begin{array}{c}\text { Casi } \\
\text { Nunca }\end{array}$ & $\begin{array}{c}\text { Casi } \\
\text { Siempre }\end{array}$ & Siempre & \\
\hline \multirow{4}{*}{$\begin{array}{l}\text { 6. ¿Considera usted, que los } \\
\text { enfoques de la enseñanza de }\end{array}$} & \multirow{2}{*}{$\begin{array}{l}\text { Algunas } \\
\text { Veces }\end{array}$} & Recuento & 8 & 3 & 8 & 3 & 22 \\
\hline & & $\begin{array}{l}\text { Recuento } \\
\text { esperado }\end{array}$ & 4,8 & 1,1 & 7,1 & 8,9 & 22,0 \\
\hline & \multirow{2}{*}{$\begin{array}{c}\text { Casi } \\
\text { Nunca }\end{array}$} & Recuento & 0 & 0 & 1 & 0 & 1 \\
\hline & & $\begin{array}{l}\text { Recuento } \\
\text { esperado }\end{array}$ & 0,2 & 0,1 & 0,3 & 0,4 & 1,0 \\
\hline \multirow{4}{*}{$\begin{array}{l}\text { principales valores en los } \\
\text { que se basa la pedagogía del } \\
\text { bachillerato internacional? }\end{array}$} & \multirow{2}{*}{$\begin{array}{c}\text { Casi } \\
\text { Siempre }\end{array}$} & Recuento & 9 & 2 & 13 & 11 & 35 \\
\hline & & $\begin{array}{l}\text { Recuento } \\
\text { esperado }\end{array}$ & 7,7 & 1,8 & 11,3 & 14,2 & 35,0 \\
\hline & \multirow[b]{2}{*}{ Siempre } & Recuento & 4 & 0 & 9 & 25 & 38 \\
\hline & & $\begin{array}{l}\text { Recuento } \\
\text { esperado }\end{array}$ & 8,3 & 2,0 & 12,3 & 15,4 & 38,0 \\
\hline \multirow{2}{*}{\multicolumn{2}{|c|}{ Total }} & Recuento & 21 & 5 & 31 & 39 & 96 \\
\hline & & $\begin{array}{l}\text { Recuento } \\
\text { esperado }\end{array}$ & 21,0 & 5,0 & 31,0 & 39,0 & 96,0 \\
\hline
\end{tabular}

Fuente: Elaboración propia 
El cálculo estadístico del chi cuadrado (Ver Tabla 3), permitió establecer que el nivel de significancia bilateral calculado es de 0.005 , es decir existe un de margen de error equivalente al 5\%, por lo que, se descarta la hipótesis nula y se acepta la hipótesis alternativa; concluyendo que: "El uso de las herramientas Web 2.0 incide en el proceso de aprendizaje de la asignatura de historia del bachillerato internacional de la población investigada".

Tabla 3. Cálculo del Chi cuadrado

\section{Pruebas de chi-cuadrado}

\begin{tabular}{lccc}
\hline & Valor & gl & $\begin{array}{c}\text { Significación } \\
\text { asintótica (bilateral) }\end{array}$ \\
\hline Chi-cuadrado de Pearson & $23,514^{\mathrm{a}}$ & 9 & 0,005 \\
\hline Razón de verosimilitud & 25,463 & 9 & 0,002 \\
\hline $\mathrm{N}$ de casos válidos & 96 & & \\
\hline a. 8 casillas (50,0\%) han esperado un recuento menor que 5. El recuento mínimo esperado es ,05. \\
\hline b. Sólo se ha calculado para una tabla 2x2
\end{tabular}

Fuente: Elaboración propia

Como se mencionó, adicionalmente se realizaron análisis de correlación de Pearson del restante de las preguntas del instrumento de investigación para dar soporte a la prueba de chi cuadrado calculado utilizando el software Orange, versión 3.24.1. donde, se pudo observar que, existen 16 correlaciones que presentan una alta correlación (alto valor de regresión) como se puede observar en la ver tabla 6. Adicionalmente, se han analizado por separado estas correlaciones para sacar posibles conclusiones de los resultados obtenidos. Para dar soporte al análisis correlacional de las preguntas, se ha adjuntado a la presente su operacionalización para que se pueda determinar el grado de relación existente entre las variables de estudio, sus dimensiones e indicadores (Ver Tabla 4 y 5$)$ :

Tabla 4. Operacionalización de la variable independiente

\begin{tabular}{|c|c|c|c|}
\hline VARIABLE & DIMENSIÓN & INDICADOR & ÍTEMS \\
\hline $\begin{array}{l}\text { La enseñanza y el aprendizaje de la } \\
\text { asignatura de historia a nivel general, } \\
\text { desarrolla en los estudiantes la }\end{array}$ & $\begin{array}{l}\text { Percepción de las } \\
\text { estrategias de } \\
\text { enseñanza } \\
\text { utilizadas por los } \\
\text { docentes }\end{array}$ & Didáctica de la enseñanza & $\begin{array}{l}\text { 1.- ¿Considera usted, } \\
\text { que el proceso de } \\
\text { enseñanza de la } \\
\text { asignatura de historia } \\
\text { utiliza estrategias } \\
\text { innovadoras? }\end{array}$ \\
\hline
\end{tabular}




\begin{tabular}{|c|c|c|c|}
\hline \multirow{5}{*}{$\begin{array}{l}\text { identidad nacional; y les permite } \\
\text { entender y comprender el desarrollo } \\
\text { de diversas culturas y lo que es } \\
\text { común a ellas; conocer la } \\
\text { interrelación entre el cambio y la } \\
\text { continuidad; desarrollar la empatía } \\
\text { histórica, es decir ver los hechos y } \\
\text { temas del pasado como fueron } \\
\text { vividos por las personas de entonces } \\
\text { a través de procesos de enseñanzas } \\
\text { desarrollados mediante un estilo de } \\
\text { aprendizaje esencialmente } \\
\text { experimental que permita reconocer } \\
\text { la diferencia entre un hecho y una } \\
\text { hipótesis, entre la realidad y la } \\
\text { ficción, entre la evidencia y la } \\
\text { afirmación, con objeto de desarrollar } \\
\text { el pensamiento crítico en los } \\
\text { ciudadanos del mañana. }\end{array}$} & $\begin{array}{l}\text { Proceso de } \\
\text { aprendizaje }\end{array}$ & $\begin{array}{l}\text { Consciente. } \\
\text { Inconsciente. }\end{array}$ & $\begin{array}{l}\text { 2.- ¿Cree usted, que } \\
\text { cuando el estudiante } \\
\text { aprende } \\
\text { conscientemente, la } \\
\text { obtención del } \\
\text { conocimiento es } \\
\text { significativo? }\end{array}$ \\
\hline & \multirow{2}{*}{$\begin{array}{l}\text { Factores que } \\
\text { intervienen en el } \\
\text { aprendizaje }\end{array}$} & Profesor /Estudiante & $\begin{array}{l}\text { 3.- ¿Considera usted, } \\
\text { que los conocimientos } \\
\text { impartidos en la } \\
\text { asignatura de historia se } \\
\text { ajustan a las necesidades } \\
\text { de aprendizaje de los } \\
\text { estudiantes? }\end{array}$ \\
\hline & & Conocimiento & $\begin{array}{l}\text { 4.- ¿Cree usted que los } \\
\text { contenidos propuestos } \\
\text { por el Bachillerato } \\
\text { Internacional para la } \\
\text { asignatura de historia } \\
\text { generan un interés en el } \\
\text { aprendizaje? }\end{array}$ \\
\hline & \multirow{2}{*}{$\begin{array}{l}\text { Enseñanza de la } \\
\text { asignatura } \\
\text { historia }\end{array}$} & Currículo & $\begin{array}{l}\text { 5.- ¿Considera usted, } \\
\text { que en el proceso de } \\
\text { enseñanza-aprendizaje } \\
\text { de historia, los } \\
\text { estudiantes alcanzan los } \\
\text { objetivos propuesto por } \\
\text { el bachillerato } \\
\text { internacional? }\end{array}$ \\
\hline & & $\begin{array}{l}\text { Evaluación. } \\
\text { Enfoque }\end{array}$ & $\begin{array}{l}\text { 6.- ¿Considera usted, } \\
\text { que los enfoques de la } \\
\text { enseñanza de historia } \\
\text { abarcan los principales } \\
\text { valores en los que se } \\
\text { basa la pedagogía del } \\
\text { bachillerato } \\
\text { internacional? }\end{array}$ \\
\hline
\end{tabular}

Tabla 5. Operacionalización de la variable dependiente

VARIABLE

\section{DIMENSIÓN}

INDICADOR

\section{Herramientas Web 2.0}

La Web 2.0 o también denominada Web colaborativa, es una Web social y participativa que permite crear, colaborar y compartir contenidos intelectuales entre todos los usuarios de la red Internet y en la que cualquiera de sus usuarios puede editar la información presentada. (Vaquerizo, Renedo, \& Valero, 2019)

Características

Asíncronas
7.- ¿Considera usted, que las herramientas de comunicación en tiempo real tales como aquellas que incorporan el chat permiten tener una información oportuna y confiable? 8.- ¿Considera usted, que el uso de los blogs, los foros $u$ otras herramientas, generan motivación en el aprendizaje de los estudiantes? 9.- ¿Recomienda usted, el

Herramientas
aplicadas en el Google Classroom
contexto del
aprendizaje
uso de aulas virtuales para desarrollar los procesos de enseñanza de la asignatura de historia? 


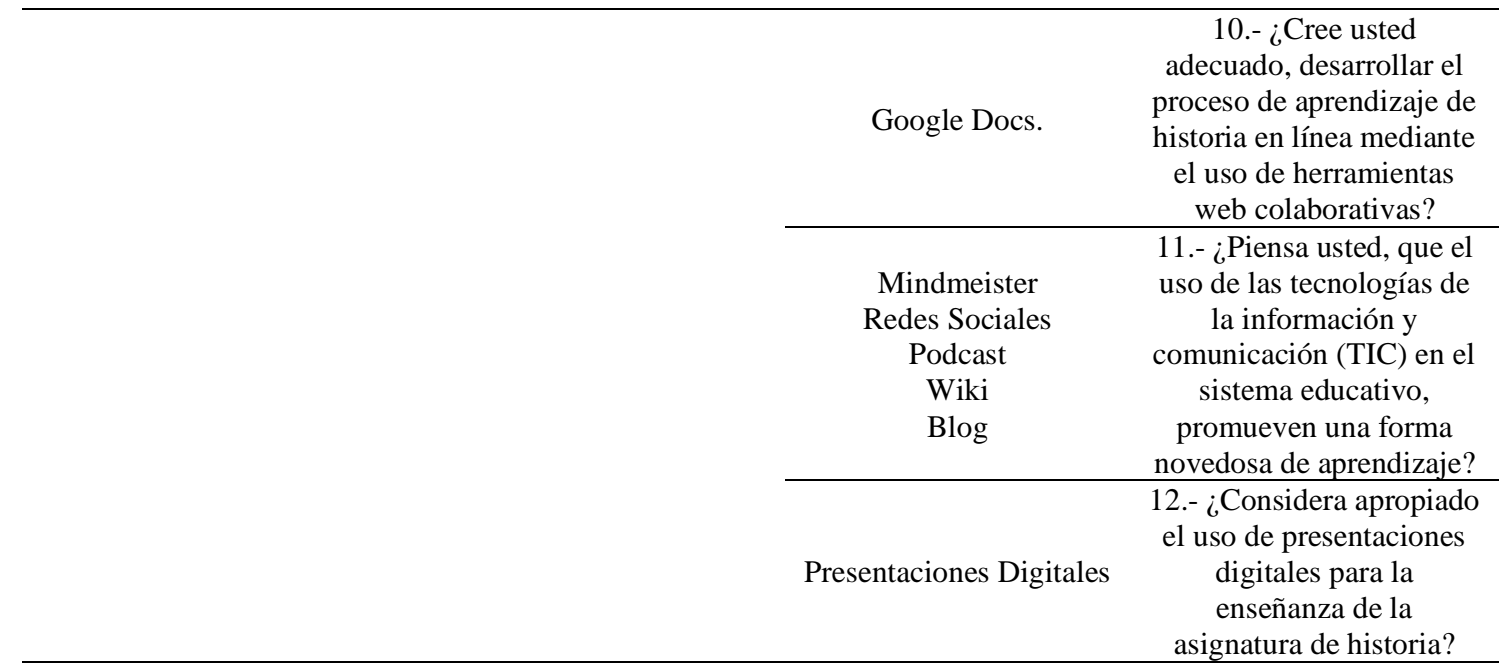

Tabla 6. Correlación de Pearson

\begin{tabular}{|c|c|c|c|}
\hline \multicolumn{4}{|c|}{ Correlación de Pearson } \\
\hline $\mathbf{N}^{\mathbf{o}}$ & $\begin{array}{c}\text { VALOR } \\
\text { "R" }\end{array}$ & VARIABLE1 & VARIABLE2 \\
\hline 1 & 0.977 & $\begin{array}{l}\text { 4. ¿Cree usted que los contenidos } \\
\text { propuestos por el Bachillerato } \\
\text { Internacional para la asignatura de } \\
\text { historia generan un interés en el } \\
\text { aprendizaje? }\end{array}$ & $\begin{array}{l}\text { 6. ¿Considera usted, que los enfoques de la } \\
\text { enseñanza de historia abarcan los principales } \\
\text { valores en los que se basa la pedagogía del } \\
\text { bachillerato internacional? }\end{array}$ \\
\hline 2 & 0.975 & $\begin{array}{l}\text { 1. ¿Considera usted, que el proceso de } \\
\text { enseñanza de la asignatura de historia } \\
\text { permite un aprendizaje consciente? }\end{array}$ & $\begin{array}{l}\text { 4. ¿Cree usted que los contenidos propuestos } \\
\text { por el Bachillerato Internacional para la } \\
\text { asignatura de historia generan un interés en } \\
\text { el aprendizaje? }\end{array}$ \\
\hline 3 & 0.973 & $\begin{array}{l}\text { 5. ¿Considera usted, que en el proceso } \\
\text { de enseñanza-aprendizaje de historia, } \\
\text { los estudiantes alcanzan los objetivos } \\
\text { propuesto por el bachillerato } \\
\text { internacional? }\end{array}$ & $\begin{array}{l}\text { 8. ¿Considera usted, que las herramientas de } \\
\text { comunicación en tiempo real tales como } \\
\text { aquellas que incorporan el chat permiten } \\
\text { tener una información oportuna y confiable? }\end{array}$ \\
\hline 4 & 0.969 & $\begin{array}{l}\text { 1. ¿Considera usted, que el proceso de } \\
\text { enseñanza de la asignatura de historia } \\
\text { permite un aprendizaje consciente? }\end{array}$ & $\begin{array}{l}\text { 6. ¿Considera usted, que los enfoques de la } \\
\text { enseñanza de historia abarcan los principales } \\
\text { valores en los que se basa la pedagogía del } \\
\text { bachillerato internacional? }\end{array}$ \\
\hline 5 & 0.967 & $\begin{array}{l}\text { 4. ¿Cree usted que los contenidos } \\
\text { propuestos por el Bachillerato } \\
\text { Internacional para la asignatura de } \\
\text { historia generan un interés en el } \\
\text { aprendizaje? }\end{array}$ & $\begin{array}{l}\text { 7. ¿Piensa usted, que el uso de las } \\
\text { tecnologías de la información y } \\
\text { comunicación (TIC) en el sistema educativo, } \\
\text { promueven una forma novedosa de } \\
\text { aprendizaje? }\end{array}$ \\
\hline 6 & 0.964 & $\begin{array}{l}\text { 1. ¿Considera usted, que el proceso de } \\
\text { enseñanza de la asignatura de historia } \\
\text { permite un aprendizaje consciente? }\end{array}$ & $\begin{array}{l}\text { 7. ¿Piensa usted, que el uso de las } \\
\text { tecnologías de la información y } \\
\text { comunicación (TIC) en el sistema educativo, } \\
\text { promueven una forma novedosa de } \\
\text { aprendizaje? }\end{array}$ \\
\hline 7 & 0.958 & $\begin{array}{l}\text { 6. ¿Considera usted, que los enfoques } \\
\text { de la enseñanza de historia abarcan los } \\
\text { principales valores en los que se basa la } \\
\text { pedagogía del bachillerato } \\
\text { internacional? }\end{array}$ & $\begin{array}{l}\text { 7. ¿Piensa usted, que el uso de las } \\
\text { tecnologías de la información y } \\
\text { comunicación (TIC) en el sistema educativo, } \\
\text { promueven una forma novedosa de } \\
\text { aprendizaje? }\end{array}$ \\
\hline
\end{tabular}


3. ¿Considera usted, que los conocimientos impartidos en la

$8 \quad 0.949$ asignatura de historia se ajustan a las necesidades de aprendizaje de los estudiantes?

3. ¿Considera usted, que los conocimientos impartidos en la

$9 \quad 0.942$ asignatura de historia se ajustan a las necesidades de aprendizaje de los estudiantes?

10. ¿Recomienda usted, el uso de aulas virtuales para desarrollar de los procesos de enseñanza de la asignatura de historia?

$10 \quad 0.938$

3. ¿Considera usted, que los conocimientos impartidos en la

$11 \quad 0.931$ asignatura de historia se ajustan a las necesidades de aprendizaje de los estudiantes?

11. ¿Cree usted adecuado, desarrollar

$12 \quad 0.921$ el proceso de aprendizaje de historia en línea mediante el uso de herramientas web colaborativas? 1. ¿Considera usted, que el proceso de

$13 \quad 0.919$
enseñanza de la asignatura de historia permite un aprendizaje consciente?

3. ¿Considera usted, que los conocimientos impartidos en la

$140.916 \quad$ asignatura de historia se ajustan a las necesidades de aprendizaje de los estudiantes?

2. ¿Cree usted, que cuando el estudiante aprende conscientemente, la

15

0.903 obtención del conocimiento es significativo?

10. ¿Recomienda usted, el uso de aulas virtuales para desarrollar de los

$16 \quad 0.902$ procesos de enseñanza de la asignatura de historia?
8. ¿Considera usted, que las herramientas de comunicación en tiempo real tales como aquellas que incorporan el chat permiten tener una información oportuna y confiable?

5. ¿Considera usted, que en el proceso de enseñanza-aprendizaje de historia, los estudiantes alcanzan los objetivos propuesto por el bachillerato internacional?

11. ¿Cree usted adecuado, desarrollar el proceso de aprendizaje de historia en línea mediante el uso de herramientas web colaborativas?

6. ¿Considera usted, que los enfoques de la enseñanza de historia abarcan los principales valores en los que se basa la pedagogía del bachillerato internacional?

8. ¿Considera usted, que las herramientas de comunicación en tiempo real tales como aquellas que incorporan el chat permiten tener una información oportuna y confiable? 2. ¿Cree usted, que cuando el estudiante aprende conscientemente, la obtención del conocimiento es significativo?

4. ¿Cree usted que los contenidos propuestos por el Bachillerato Internacional para la asignatura de historia generan un interés en el aprendizaje?

7. ¿Piensa usted, que el uso de las tecnologías de la información y comunicación (TIC) en el sistema educativo, promueven una forma novedosa de aprendizaje?

8. ¿Considera usted, que las herramientas de comunicación en tiempo real tales como aquellas que incorporan el chat permiten tener una información oportuna y confiable?

Fuente: Elaboración propia

Correlación de la $1^{\circ}$ y $4^{\circ}$ pregunta:

Según estudio de la correlación de la pregunta $n^{\circ} 1$ y $n^{\circ} 4$, se puede concluir que existe una relación estadísticamente significativamente $(\mathrm{r}=0.97)$ entre el proceso de enseñanza de la asignatura de historia y los contenidos propuestos por el bachillerato internacional, los cuales generan un interés en el aprendizaje adquirido conscientemente. (Ver figura 5) 


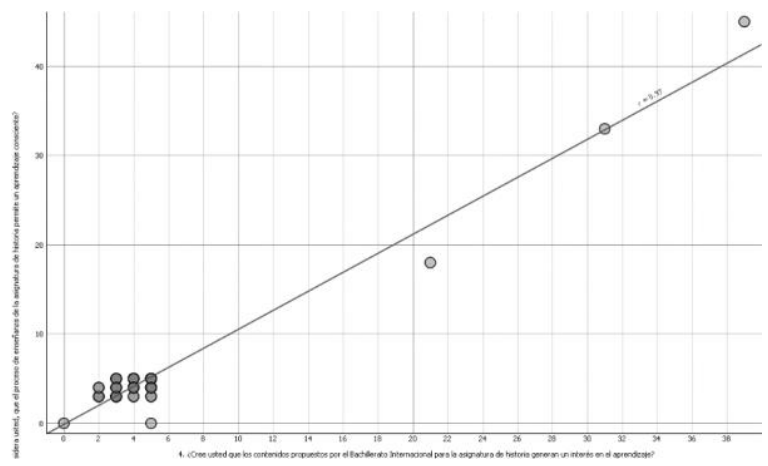

Figura 4. Análisis correlacional de Pearson (pregunta 1 y 4 ) Fuente: Elaboración propia

Correlación de la $1^{\mathrm{o}}$ y $6^{\mathrm{o}}$ pregunta:

Según el estudio de correlación de la pregunta $\mathrm{n}^{\circ} 1$ y $\mathrm{n}^{\circ} 6$, se puede deducir que existe una relación estadísticamente significativa $(r=0.97)$ entre el proceso de enseñanza de la asignatura de historia a través de un aprendizaje consciente y los principales valores en los que se basa el enfoque pedagógico del bachillerato internacional. (ver figura 6)

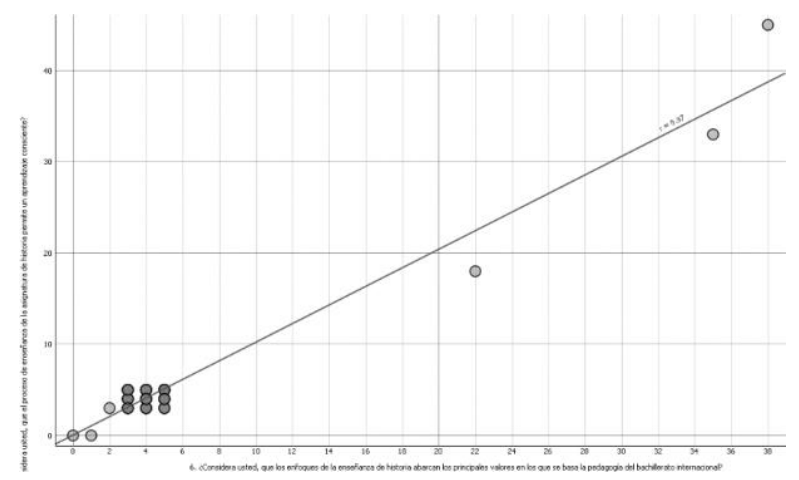

Figura 5. Análisis correlacional de Pearson (pregunta 1 y 6 ) Fuente: Elaboración propia

Correlación de la $1^{\circ}$ y $7^{\circ}$ pregunta:

Según el análisis de estudio de la correlación de la pregunta $\mathrm{n}^{\circ} 1 \mathrm{y} \mathrm{n}^{\circ} 7$, se puede deducir que existe una relación estadísticamente significativa $(\mathrm{r}=0.96)$; considerando que, el proceso de enseñanza de la asignatura de historia y el uso de las tecnologías de la información y comunicación promueven formas novedosas de aprender más aun cuando se lo hace de manera consciente. (ver figura 7) 


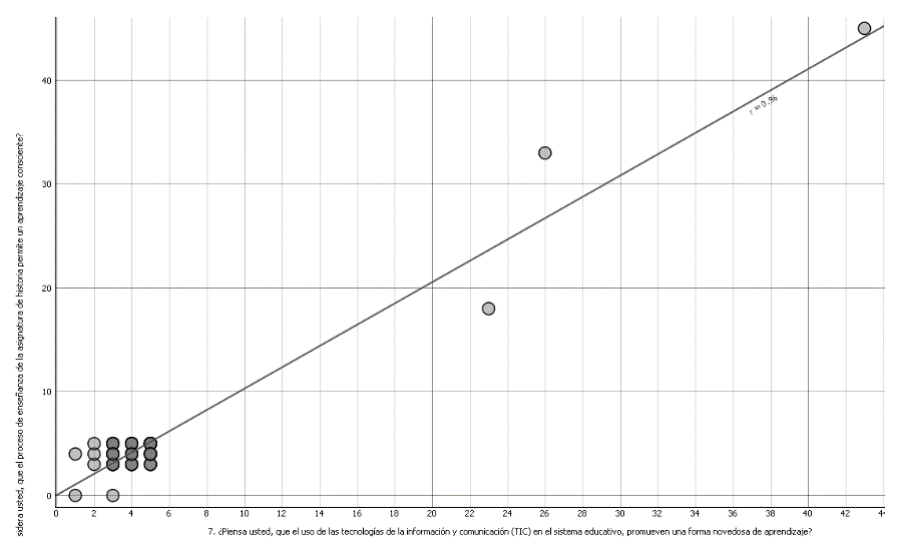

Figura 6. Análisis correlacional de Pearson (pregunta 1 y 7). Fuente: Elaboración propia

Correlación de la $4^{\circ}$ y $7^{\circ}$ pregunta:

Según el análisis de la correlación entre las preguntas $n^{\circ} 4$ y n ${ }^{\circ} 7$, se puede deducir que existe una relación estadísticamente significativa $(r=0.97)$ entre el uso de las tecnologías de la información y comunicación en el sistema educativo y los contenidos propuesto por el bachillerato internacional para la asignatura de historia donde se promueve una forma novedosa de aprender generando a su vez un interés en dicho aprendizaje. (ver figura 8)

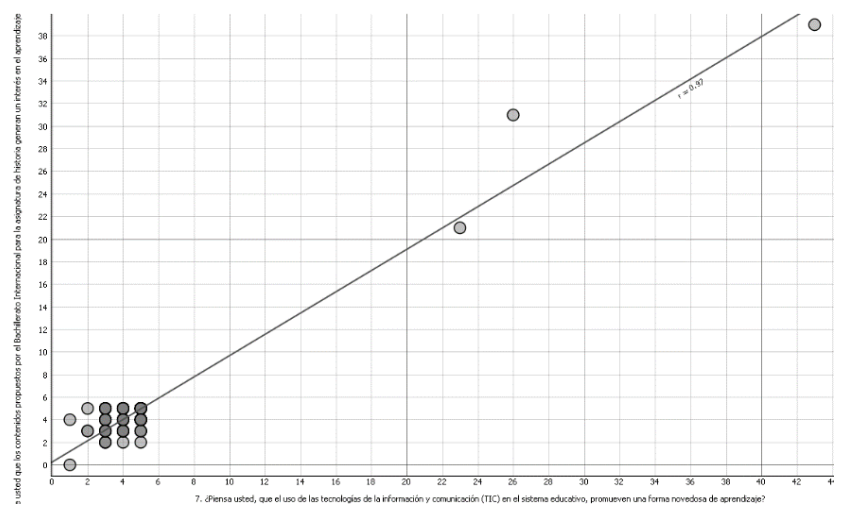

Figura 7. Análisis correlacional de Pearson (pregunta 4 y 7). Fuente: Elaboración propia

Correlación de la $5^{\circ}$ y $8^{\circ}$ pregunta:

Según el estudio de correlación de la pregunta $n^{\circ} 5 \mathrm{y} \mathrm{n}^{\circ} 8$, se puede deducir que existe una relación estadísticamente significativa $(r=0.97)$ entre los procesos de enseñanza-aprendizaje de la asignatura de historia y las herramientas de comunicación en tiempo real utilizadas, donde se obtiene información oportuna y confiable y de esta manera, alcanzar los objetivos propuestos por el bachillerato internacional. (ver figura 9) 


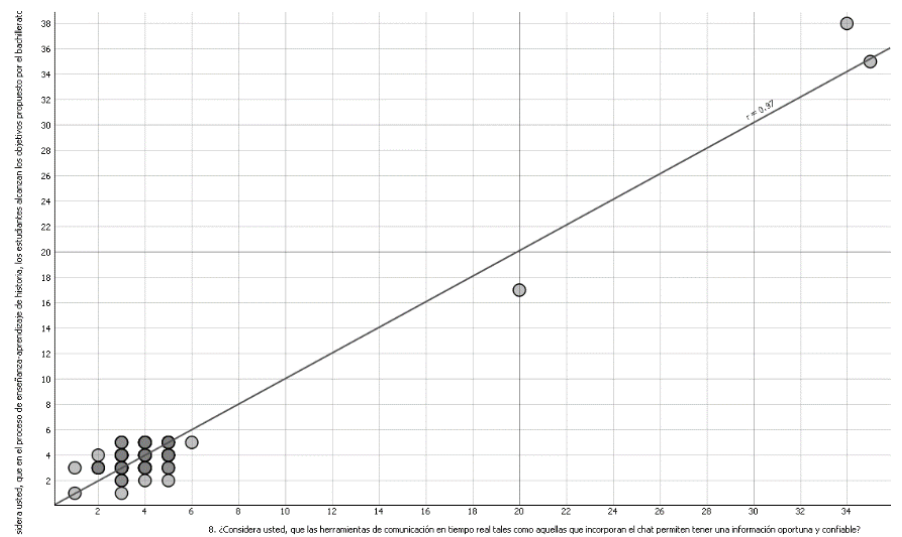

Figura 8. Análisis correlacional de Pearson (pregunta 5 y 8). Fuente: Elaboración propia

Correlación de la $6^{\circ}$ y $7^{\circ}$ pregunta:

Según el estudio de correlación de la pregunta $\mathrm{n}^{\circ} 6 \mathrm{y} \mathrm{n}^{\mathrm{o}} 7$, se puede deducir que existe una relación estadísticamente significativa $(r=0.96)$ entre los enfoques en la enseñanza de historia basados en la pedagogía del bachillerato internacional y el uso de las tecnologías de la información y comunicación donde se promueve una novedosa forma de aprendizaje. (ver figura 10)

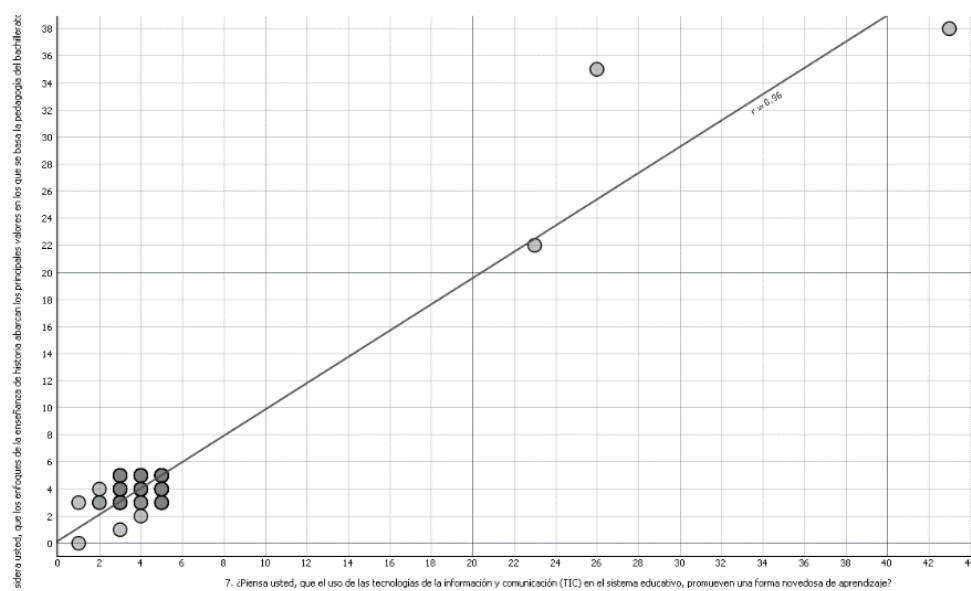

Figura 9. Análisis correlacional de Pearson (pregunta 6 y 7). Fuente: Elaboración propia

\section{Discusión y conclusiones}

La investigación realizada por Traverso y otros autores en el año 2013, presenta una perspectiva muy clara sobre el uso de las herramientas web 2.0 como una alternativa para proceso de enseñanza y aprendizaje bajo una perspectiva constructivista. Una de las cualidades más destacables en la incorporación de estas aplicaciones en el entorno 
educativo, es que facilitan el trabajo en equipo, la solución de problemas y la toma de decisiones de forma conjunta. Bajo este enfoque, la participación en comunidades virtuales puede proporcionar a docentes y estudiantes una oportunidad para crear colectivos de aprendizaje virtual (Traverso, y otros 2013, 5,6).

Los tiempos actuales demandan la modificación de las prácticas educativas establecidas como comunes entre los docentes, es por eso por lo que, es imposible en este contexto (COVID-19), deslindar el uso de estas herramientas web en los procesos de enseñanza establecidos por los docentes en su diseño curricular, y en las prácticas derivadas del proceso de aprendizaje determinado por los estudiantes consumidores de estos recursos y herramientas. Se debe considerar además que la tecnología por sí sola, no puede fundamentar ni generar aprendizajes, se necesita de la guía y acompañamiento docente el cual, tiene el rol fundamental de motivar al estudiante en el uso y descubrimiento de estas y otras herramientas que le permitan mejorar sus competencias académicas y su rendimiento (Villoria 2009, 15).

A través de esta investigación se ha podido confirmar la hipótesis afirmativa planteada en la población de estudio que demuestra que el uso de las herramientas web 2.0, influyen en el proceso de aprendizaje de los estudiantes de bachillerato internacional de la Unidad Educativa Fiscomisional "Juan XXIII" en la asignatura de Historia, considerando además que, su uso debe ser una prioridad por parte de los docentes en el diseño curricular de las clases impartidas para que los estudiantes puedan profundizar los conocimientos adquiridos (Alejo y Sánchez 2020, 25) (Bolívar y Pedraza 2019, 166).

Las herramientas web 2.0 más utilizadas por los estudiantes del bachillerato internacional para el aprendizaje de la asignatura de historia son: Chats, foros, wikis, entre otras, que se encuentran presentes en plataformas educativas y Entornos Virtuales de aprendizaje (EVA) como: Google Classroom, Moodle y Google Docs. La utilización de estas herramientas, recursos y plataformas, permiten además el trabajo colaborativo y una comunicación sincrónica y asincrónica con el docente de esta signatura lo que facilita el diseño curricular de las clases impartidas y el uso de herramientas tecnológicas y competencias digitales por parte de los estudiantes (Summuer 2018, 32). Los estudiantes a través del uso de estos recursos y plataformas manifiestan de manera empírica una motivación intrínseca expresada en la utilización de recursos digitales derivados de las herramientas web 2.0 que les permiten aprender de manera independiente y colaborativa. (Bolívar y Pedraza 2019, 166). 
Se sugiere que esta investigación pueda servir como punto de partida para futuras reflexiones sobre la influencia del uso de las herramientas tecnológicas en el ámbito educativo y que permita mejorar los procesos de enseñanza y aprendizaje no solo en el nivel bachillerato en la asignatura de historia, sino en los otros subniveles y niveles establecidos en el sistema educativo.

\section{Referencias}

Abad, Luis David. Las herramientas de la web 2.0. 2018. https://sites.google.com/site/web20lasherramientas/caracteristicas-de-laherramientas-de-la-web-2-0.

Aithal, P, y Suresh Kumar. «Teaching-Leraning Process in Higher Education Intitutions.» International Journal of Multidisciplinary Research and Modern Education (IJMRME), 2016: 663-671.

Alejo, Lizeth, y Lady Sánchez . «Herramientas Web 2.0 en el proceso de enseñanzaaprendizaje.» Repositorio de la Universidad San Ignacio de Loyola. 2020. 25-27.

Andalucía. «Revista Digital para profesionales de la enseñanza.» Aprendizaje: Definición, factores y clases, 2008: 1-6.

Ávila, William Darío. «Hacia una reflexion Historica de las TIC.» Hallazgos, 2013: 215230.

Belloch, Consuelo . «Las Tecnologías de la Información y Comunicación en el Aprendizaje.» DeptoMIDE. Universidad de Valencia. 2012. 1-9.

Bolívar, Patricia, y Milagros Pedraza. «Incidencia de las herramientas WEB 2.0 en el aprendizaje de la lengua castellana en los estudiantes de la básica secundaria.» 2019. 50-61,166-168.

Bonilla, Francy Milena, y Diana Siomara Cubillos. Evolución de la historia de las TIC. Febrero de 2012. https://sites.google.com/site/ticsyopal5/assignments/homeworkforweekofoctober 18 th.

Calleja, Javier. «ABP y el uso de las TIC: Una propuesta para la mejora de la educación.» 2016. 5-25,99-101.

Cela, Karina, Walter Fuentes, Catalina Alonso, y Franklin Sánchez. «Evaluación de herrameintas WEB 2.0, estilos de aprendizaje.» Estilos de Aprendizaje, 04 2010: 117-123.

Chen, Caterina. Significado de TIC (Tecnologías de la información y la comunicación). 21 de 05 de 2019. https://www.significados.com/tic/.

Chumpik, Tukup. «Herramientas Web 2.0 Para El Aprendizaje de la División en Quinto año de Educación Básica.» Febrero de 2020. 10-17,62-63.

Constitucion de la República del Ecuador. 2008.

Echeng, Razep, y Abel Usoro. «Enhancing the use of Web 2.0 Technologies in Higher Education: Students' and Lectures' Views Students' and Lectures' View.» Journal of International Technology and Information Management Journal of International Technology and Information Management, 2016: 92-103. 
Emma, Emilie . Monografias.com. 17 de Noviembre de 2009. https://www.monografias.com/trabajos76/proceso-ensenanza-aprendizajehistoria/proceso-ensenanza-aprendizaje-historia.shtml.

Ferro Soto, Carlos, Ana Isabel Martínez Senra, y MaCarmen Otero Neira. «Ventajas del uso de las TICs en el proceso de enseñanza-aprendizaje desde la óptica de los docentes universitarios españoles.» Edutec. Revista Electronica de Tecnología Educativa., 07 2009: 2-9.

Harris, Albert, y Alan Rea. «Web 2.0 and Virtual World Technologies: A Growing Impact on IS Education.» Journal of Information Systems Education, Vol. 20(2), 2019: 137-143.

Hernández, Roberto, Collado Fernández , y Maria del Pilar Baptista. «Metodología de la investigación.» México D.F.: McGRAW-HILL / INTERAMERICANA EDITORES, S.A. DE C.V., 2010. 33-356, 593-601.

Hernández, Verónica. e-Leraning Master. 20 de Abril de 2018. http://elearningmasters.galileo.edu/2018/04/20/trabajo-colaborativo/.

Latorre, Marino. «Universidad Marcelino Champagnat.» Historia de las WEB, 1.0, 2.0, 3.0 y 4.0. 2018. $1-8$.

Ley Orgánica de Educación Intercultural. Marzo de 2011.

Ley Orgánica de Telecomunicaciones. 2015.

López, Juan José. Caracteristicas principales de la WEB 2.0. 2014. https://comunidad.iebschool.com/elearning/2014/12/12/caracteristicasprincipales-de-la-web-2-0/.

Maldonado, Jorge. «Metodología de la Investigación social: Paradigmas: cuantitativo, sociocrítico, cualitativo, complementario.» Ediciones de la U, 2018. 19-22,67-77.

Marino, Roberto. Conectados en el ciberespacio. Noviembre de 2010. https://fuerzaprofesional.wordpress.com/tecnologia-de-la-informacion-y-de-lacomunicacion-tic/.

Márquez, Mayra Verónica. «Implementación de un entorno formativo multimedia basado en herramientas web 2.0 interactivas, para mejorar el proceso enseñanza aprendizaje en el área de estudios sociales.» 8 de 2013. 9-25, 66-67.

Milena, Bonilla Amado Francy, y Cubillos Ospina Diana Siomara. «Evolucion de la historia de las TICs.» Tecnología de la información y comunicación - YOPAL. $\begin{array}{ll}\text { febrero de } 2012 . & \end{array}$ https://sites.google.com/site/ticsyopal5/assignments/homeworkforweekofoctober 18 th.

Ministerio de Educación del Ecuador. ACUERDO Nro. MINEDUC-MINEDUC-201800081-A. 2018.

Ministerio de Telecomunicaciones y de la Sociedad de la Información. «Plan Nacional de Telecomunicaciones y Tecnologias de la Informacion del Ecuador 2016-2021.» 2015. 25-35.

Naciones Unidas. «America Latina y las nuevas tecnologías.» Los derechos de la infancia en la era del Internet. Septiembre de 2014. 12-15.

Organización de Bachillerato Internacional. «Guia Historia primera evaluacion 2017.» 2017. 6-14,77-100. 
Organización de las Naciones Unidas para la Educación la Ciencia y la Cultura. «Enfoques estratégicos sobre las TICS en Educación en América Latina y el Caribe.» 2013: 13-47.

Pérez. Madame de La Fayette. 2016. https://madamedelafayette.wordpress.com/cualesson-las-caracteristicas-de-las-tics/.

Pérez, Julián, y Ana Gardey. Definición.de. 2013. https://definicion.de/proceso-deaprendizaje/.

Rautinainen, Matti, Eija Räikkönen, Anna Veijola, y Simo Mikkonen. «History teaching in Finnish general upper secondary schools: Objectives and practices.» History Education Research Journal, 16(2), 2019: 291-304.

Robles, Pilar, y Manuela Rojas. «alidation by expert judgements: two cases of qualitative research in Applied Linguistics.» Revista Nebrija de Linguistica Aplicada a la Enseñanza de Las Lenguas, 2015: 1-10.

Rodriguez, Lizbeth. «Evaluación Formativa en la asignatura de Historia del Programa del Diploma del Bachillerato Internacional en la Unidad Educativa Maurice Ravel de la ciudad de Quito, en el período 2019.»09 de 2019. 40-59,111-119.

Rodriguez, Martha. Importancia de las TICs en la educación. 17 de 07 de 2009. http://ticsenlaeducacion-yaneth.blogspot.com/.

Ruiz. Psicología y Mente. 2018. https://psicologiaymente.com/miscelanea/alfa-decronbach.

Ruiz, Franscisco José. «Web 2.0. un nuevo entorno de aprendizaje en la red.» Revista DIM, 2009: 1-7.

Sampedro, Begoña E., y Verónica Marin. «Conocimiento de los futuros educadores sociales de las herramientas WEB 2.0.» Pixel-Bit Revista de Medios y Educación. $N^{o}$ 47, 2015: 41-58.

Sandoval. «e-Learning Master.» Herramientas web 2.0 para profesores activos y $\begin{array}{llllll}\text { creativos. } & 10 & \text { de } & \text { Mayo } & \text { de } & 2017 .\end{array}$ http://elearningmasters.galileo.edu/2017/05/10/herramientas-web-2-0-paraprofesores-activos-y-creativos/ (último acceso: 4 de Mayo de 2020).

- Enseñanza Virtual, Recursos y herramientas. 13 de diciembre de 2016. http://elearningmasters.galileo.edu/2016/12/13/7-tipos-de-herramientas-web-2$0 \%$

Suarez, Mario. Coeficiente de correlación de Pearson para datos agrupados en $\begin{array}{llllll}\text { intervalos. } & 07 & \text { de } & \text { junio }\end{array}$ https://www.monografias.com/trabajos86/correlacion-pearson-datos-agrupadosintervalos/correlacion-pearson-datos-agrupados-intervalos.shtml.

Summuer, Evren. «Factors related to college students' self-directed learning with technology.» AJET - Australasian Journal of Educational Technology, Septiembre 2018: 29-39.

Tonon, Graciela. «La utilización del método comparativo en estudios cualitativos en ciencia politica y ciencias sociales.» KAIROS-Revista de temas sociales, 2011: 111.

Traverso, Hugo Emilio, Laura Beatriz Prato, Liliana Noemí Villoria, y Gustavo Alfredo Gomez. «Herramientas de la Web aplicadas a la educación.» 2013. 1-8. 
O人ıвьu Wimblu, Rev. Estud. de Psicología UCR, 16(1) 2021 (Enero-Junio): 9-28 /ISSN: 1659-2107

Vaquerizo, Belen, Eduardo Renedo, y Miguel Valero. «Aprendizaje colaborativo en grupo: Herramientas Web 2.0.» Jornadas de Enseñanza Universitaria de la Informática, 2019: 447-450.

Vilà, Ruth. «¿Cómo hacer un análisis cuantitativo de datos de tipo descriptivo.» 6 de Junio de 2006. 1-6.

Villoria, Noemi. «Aplicaciones Web 2.0.» 2009. 13-17.

Yanez, Patricio. «El proceso de aprendizajefases y elementos fundamentales.» Revista San Gregorio (Dialnet Plus), no 11 (2016): 72-79. 